\title{
Myasthenia Gravis Presenting Initially as Acute Respiratory Failure
}

\author{
Seung Jun Lee MD, Jaehyung Hur MD, Tae Won Lee MD, Sunmi Ju MD, \\ Seung Hun Lee MD, Ki-Jong Park MD, Yu Ji Cho MD, Yi Yeong Jeong MD, \\ Jong Deog Lee MD, and Ho Cheol Kim MD
}

\begin{abstract}
Introduction
Myasthenia gravis (MG) is an autoimmune disorder of neuromuscular transmission, characterized by fluctuating weakness in certain skeletal muscles. ${ }^{1}$ The reported prevalence of MG (5-20/100,000 individuals) has been continually increasing. ${ }^{2-5}$ The clinical courses of patients with MG are quite diverse, from complete remission to MGrelated death. ${ }^{6} \mathrm{MG}$ crisis, defined as an exacerbation of MG requiring ventilatory assistance, is a potentially fatal complication of MG. ${ }^{7}$ MG crisis occurs in $\sim 20 \%$ of patients with $\mathrm{MG}$ and usually during the first $2 \mathrm{y}$ of illness. ${ }^{8,9}$ Weakness in certain skeletal muscles, including ocular, bulbar, and limb muscles, is typically the initial symptom of MG. However, MG crisis can be the initial presentation in some patients on rare occasions. Here, we report a patient with MG initially presenting with acute respiratory failure, triggered by a gastrointestinal infection.
\end{abstract}

\section{Case Summary}

A 77-y-old man was referred to the pulmonary clinic because of acute respiratory failure on the day of admission. The patient presented to the emergency department with vomiting and diarrhea ( $>10$ occurrences) after ingesting raw oysters the previous day and was admitted for treatment of acute gastroenteritis. On the day of admission, sudden respiratory failure developed immediately af-

Drs SJ Lee, Hur, TW Lee, Ju, SH Lee, Cho, Jeong, JD Lee, and Kim are affiliated with the Division of Pulmonology and Allergy, Department of Internal Medicine, and Dr Park is affiliated with the Department of Neurology, College of Medicine, Gyeongsang National University, Jinju, Korea.

The authors have disclosed no conflicts of interest.

Correspondence: Ho Cheol Kim MD, Department of Internal Medicine, Gyeongsang National University School of Medicine, 15 Jinju-daero 816beon-gil, Jinju, Gyeongsangnam-Do 660-751, Korea. E-mail: hochkim@empal.com.

DOI: $10.4187 /$ respcare. 03210 ter he felt dyspnea. He had no comorbid disease except hypertension. He was a never-smoker and did not drink alcohol.

On physical examination during mechanical ventilation, he was alert and oriented. On neurologic examination, his motor and sensory tests were normal with a brisk deep tendon reflex. There was no evidence of pathologic reflex. Cranial nerve examination showed that light reflex was prompt and ocular movement was full without a ptosis. His vital signs were as follows: blood pressure, 150/80 $\mathrm{mm} \mathrm{Hg}$; pulse, 92 beats/min; breathing frequency, 20 breaths/min; and oxygen saturation, $99 \%$ on pressure support mechanical ventilation with a PEEP of $5 \mathrm{~cm} \mathrm{H}_{2} \mathrm{O}$, pressure support of $8 \mathrm{~cm} \mathrm{H}_{2} \mathrm{O}$, and $\mathrm{F}_{\mathrm{IO}_{2}}$ of 0.4 .

His initial white blood cell count was 13,150 cells $/ \mu \mathrm{L}$, with hemoglobin at $14.6 \mathrm{~g} / \mathrm{dL}$ and a platelet count of 252,000 cells $/ \mu \mathrm{L}$. The level of serum C-reactive protein was $14.85 \mathrm{mg} / \mathrm{L}$. There was no definite abnormality on chest radiography (Fig. 1). Analysis of arterial blood gas when he felt dyspnea just before applying the mechanical ventilator showed a pH of $7.19, \mathrm{P}_{\mathrm{aCO}_{2}}$ of $71 \mathrm{~mm} \mathrm{Hg}, \mathrm{P}_{\mathrm{aO}_{2}}$ of $57 \mathrm{~mm} \mathrm{Hg}$, and oxygen saturation of $81.9 \%$ on room air. A computed tomography (CT) scan of the brain was performed because type II respiratory failure as a result of alveolar hypoventilation was suspected. However, there was no abnormal lesion in the central nervous system. There seemed little likelihood that the patient had abnormal function of the respiratory system, considering his chest radiography, lack of smoking history, and past medical history. Paralytic shellfish poisoning by ingestion of raw oysters was our initial clinical diagnosis. Weaning from mechanical ventilation was attempted repeatedly on $>5$ consecutive d. However, hypercapnic acidosis occurred at every weaning attempt with no improvement. A chest $\mathrm{CT}$ was performed to further evaluate his respiratory system, and there was segmental atelectasis in both lower lobes. Incidentally, a $1.6-\mathrm{cm}$ soft tissue mass, supposedly a thymoma, was detected in the anterior mediastinum (Fig. 2).

Considering the clinical course and chest CT findings, MG was suspected clinically. His serum level of acetyl- 


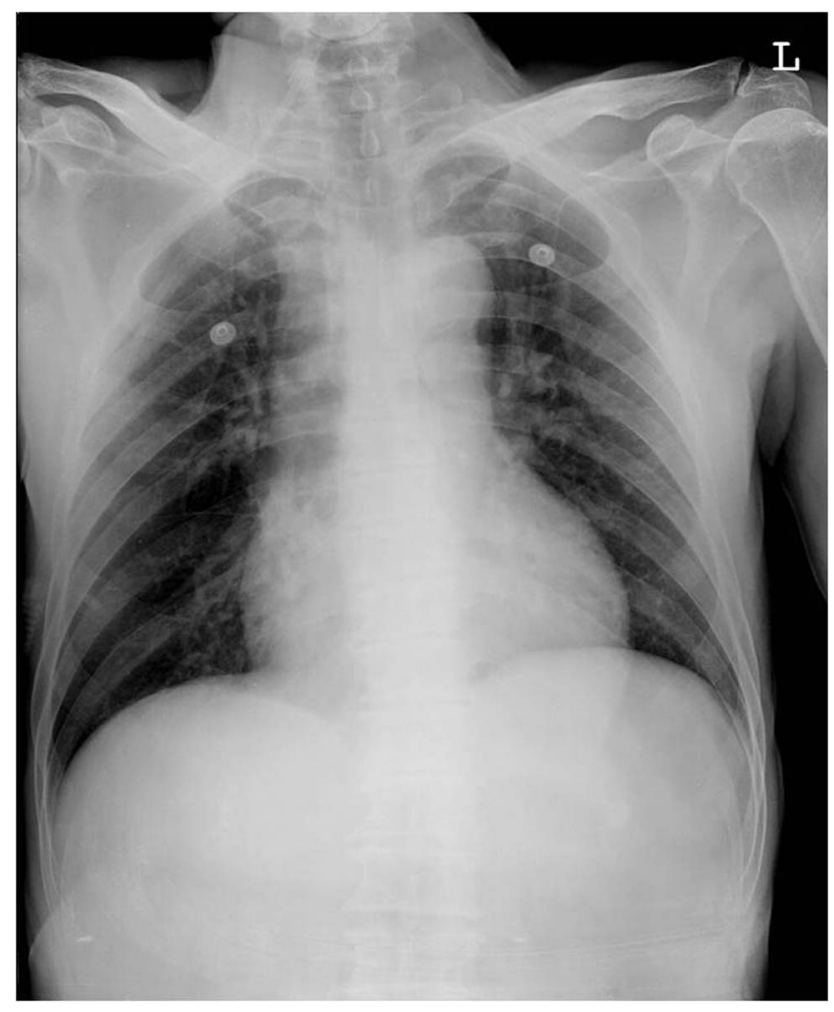

Fig. 1. Initial chest radiography showing no definite abnormality.

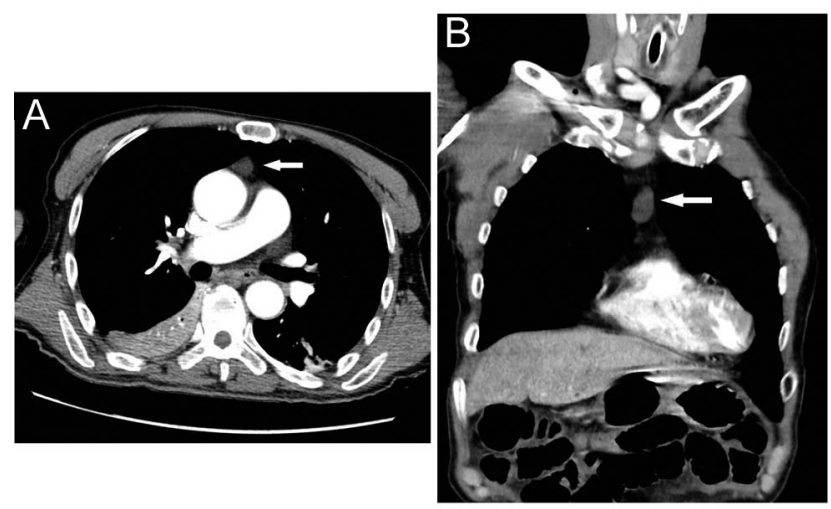

Fig. 2. Chest computed tomography showing right side-dominant atelectasis in both lower lobes with no obstructive cause and an anterior mediastinal mass (arrows). A: Transverse view. B: Coronal view.

choline receptor-binding antibodies was $0.215 \mathrm{nmol} / \mathrm{L}$, and that of the acetylcholine receptor-blocking antibody was $<15.0 \mathrm{nmol} / \mathrm{L}$. A nerve conduction study showed a normal range of amplitude of compound muscle action potential, terminal latency, conduction velocity, and late response on the motor and sensory nerves in all extremities. Repetitive nerve stimulation testing on the right first dorsal interossei and abductor digiti minimi muscles showed a normal resting compound muscle action potential am-

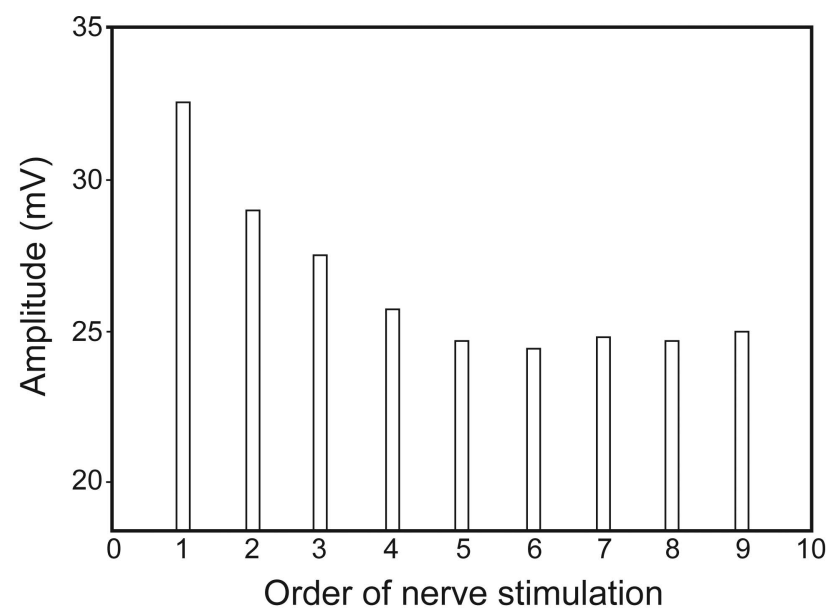

Fig. 3. Repetitive nerve stimulation at $2 \mathrm{~Hz}$ on the right first dorsal interossei muscle showing an $18.9 \%$ decrease in amplitude of compound muscle action potential from the first to the fifth stimulation (32.7 vs $24.7 \mathrm{mV}$ ).

plitude and a significant decrease both at $2-5$ and $50 \mathrm{~Hz}$ stimulation (Fig. 3), confirming the diagnosis of MG.

Methylprednisolone at $1,000 \mathrm{mg} / \mathrm{d}$ for $3 \mathrm{~d}$ followed by $60 \mathrm{mg} / \mathrm{d}$ and $180 \mathrm{mg} / \mathrm{d}$ pyridostigmine bromide were administered. The patient was successfully weaned from mechanical ventilation and extubated on the third $d$ after administration of methylprednisolone and pyridostigmine. He was discharged from the hospital, and an elective thymectomy was scheduled.

\section{Discussion}

The current case report demonstrates that acute respiratory failure can be an initial presentation of MG. Patients with MG usually present with repeated and fluctuating weakness of specific skeletal muscles. The weakness typically worsens with prolonged activity and improves with rest. ${ }^{7}$ Isolated ocular muscle involvement is the most common presentation of MG, but weakness can also arise in bulbar or other muscles. There are few reports of acute respiratory failure as an initial presentation of MG. ${ }^{10,11}$ The progression of respiratory failure in this case was much more acute than that of previous reports. Dyspnea developed $6 \mathrm{~h}$ after hospital admission, and mechanical ventilation was needed immediately after that.

Our initial presumed diagnosis was acute gastroenteritis and paralytic shellfish poisoning-related respiratory failure. We wanted to measure the shellfish toxin levels in the patient's urine, but the test was unavailable at our institution. Because paralytic shellfish poisoning is a self-limited disease, ${ }^{12}$ we repeatedly attempted to wean the patient from the mechanical ventilator in anticipation of improvement. However, all weaning attempts failed, and paralytic 


\section{Myasthenia Gravis Presenting as Acute Respiratory Failure}

shellfish poisoning did not seem to be the cause of respiratory failure in our patient.

The serum levels of acetylcholine receptor-binding and acetylcholine receptor-blocking antibodies were normal in our case. Acetylcholine receptor antibodies are detected in $55-70 \%$ of patients with ocular $\mathrm{MG}$ and in $80-90 \%$ of patients with generalized MG. ${ }^{13}$ Even though the levels of other autoantibodies, including muscle-specific kinase, anti-striated muscle, and skeletal muscle titin antibodies, were not determined, an electromyogram confirmed the diagnosis of MG. Furthermore, there were marked responses to corticosteroid and pyridostigmine, including prompt weaning from mechanical ventilation and subsequent extubation, reconfirming the diagnosis of $\mathrm{MG}$.

A chest CT scan is mandatory in patients diagnosed with MG. ${ }^{13}$ In this case, the chest CT scan was performed before the diagnosis of MG. Bilateral passive atelectasis was an anticipated finding. An anterior mediastinal mass was detected coincidently, which raised the possibility of MG. The thymus is a specialized organ of the immune system. It generates many $\mathrm{T}$ lymphocytes, which play important roles in the immune system. Thymic abnormalities, including hyperplasia and thymoma, are present in $\sim 80 \%$ of patients with MG. ${ }^{14}$ This case indicates that a thymic abnormality can be a clinical clue to aid in the diagnosis of MG when the clinical presentation is atypical.

\section{Teaching Points}

- Patients with MG can initially present with acute respiratory failure.

- Respiratory failure as an initial presentation of MG can occur very acutely, within $6 \mathrm{~h}$.

- Although respiratory infection such as pneumonia is the most common triggering factor, gastroenteritis is one of the uncommon triggering factors of an MG crisis.

- If hypercapnic respiratory failure occurs in patients with no underlying pulmonary disease and the patient does not improve despite appropriate management, physicians should consider performing diagnostic tests for MG.

- A negative result for the acetylcholine receptor antibody does not rule out the diagnosis of $\mathrm{MG}$.

\section{REFERENCES}

1. Drachman DB. Myasthenia gravis. N Engl J Med 1994;330(25): 1797-1810.

2. Phillips LH. The epidemiology of myasthenia gravis. Semin Neurol 2004;24(1):17-20.

3. Phillips LH 2nd. The epidemiology of myasthenia gravis. Ann NY Acad Sci 2003;998:407-412.

4. Phillips LH 2nd, Torner JC. Epidemiologic evidence for a changing natural history of myasthenia gravis. Neurology 1996;47(5):12331238.

5. Isbister CM, Mackenzie PJ, Anderson D, Wade NK, Oger J. Cooccurrence of multiple sclerosis and myasthenia gravis in British Columbia. Mult Scler 2003;9(6):550-553.

6. Oosterhuis HJ. The natural course of myasthenia gravis: a long term follow up study. J Neurol Neurosurg Psychiatry 1989;52(10):11211127.

7. Bershad EM, Feen ES, Suarez JI. Myasthenia gravis crisis. South Med J 2008;101(1):63-69.

8. Sellman MS, Mayer RF. Treatment of myasthenic crisis in late life. South Med J 1985;78(10):1208-1210.

9. Thomas CE, Mayer SA, Gungor Y, Swarup R, Webster EA, Chang I, et al. Myasthenic crisis: clinical features, mortality, complications, and risk factors for prolonged intubation. Neurology 1997;48(5): 1253-1260.

10. Vaidya H. Case of the month: unusual presentation of myasthenia gravis with acute respiratory failure in the emergency room. Emerg Med J 2006;23(5):410-413.

11. Kim WH, Kim JH, Kim EK, Yun SP, Kim KK, Kim WC, Jeong HC. Myasthenia gravis presenting as isolated respiratory failure: a case report. Korean J Intern Med 2010;25(1):101-104.

12. Etheridge SM. Paralytic shellfish poisoning: seafood safety and human health perspectives. Toxicon 2010;56(2):108-122.

13. Juel VC, Massey JM. Myasthenia gravis. Orphanet J Rare Dis 2007; 2:44.

14. Cavalcante P, Le Panse R, Berrih-Aknin S, Maggi L, Antozzi C, Baggi $F$, et al. The thymus in myasthenia gravis: site of "innate autoimmunity"? Muscle Nerve 2011;44(4):467-484. 